\title{
Delayed Presentation of Acute Coronary Syndrome: A Challenge in its Early Management
}

\author{
Acharya P, ${ }^{1}$ Adhikari RR, ' Bhattarai J,' Shrestha NR, ${ }^{1}$ Sharma SK, ' Karki P' \\ 'Department of Internal Medicine, B.P. Koirala Institute of Health Sciences, Dharan, Nepal.
}

\begin{abstract}
Introduction: The time of presentation of acute coronary syndrome from the onset of chest pain determines the treatment modality and prognosis. Delayed presentation is associated with a poor outcome. In the present study, we tried to find out the causes of late presentation of ACS in a tertiary care center in the eastern part of Nepal.
\end{abstract}

Methods: It was a cross-sectional descriptive study that included 100 consecutive patients with ACS presenting to our institute over a period of 8 months. They were studied for their demographic profile, delay in presentation, the management done at the local centers and their final diagnosis.

Results: We found that patients living within Dharan City reached BPKIHS within 20 hours of the onset of chest pain while those from outside the city who came directly reached within 63 hours. Other patients reached their respective local centers (health posts, district hospitals and private clinics) within 39 hours. The commonest cause of delay was vehicular problem followed by unnecessary delay at the local centers. The work up for chest pain was inadequate in these centers. Late presentation to our institute significantly affected the optimal management.

Conclusions: We found that significant number of patients with ACS from eastern Nepal presented late in our tertiary care center. In order to improve ACS outcome in this region, we advise equipping the local centers with electrocardiogram machines, improvement in ambulance services and a greater emphasis on coronary artery disease awareness programs as well as initiating preventive measures.

Key words: acute coronary syndrome, chest pain, delayed presentation, electrocardiogram

\section{INTRODUCTION}

Non-communicable diseases have surpassed infectious diseases as the major cause of morbidity and mortality in the developed as well as the developing world except sub-Saharan Africa. ${ }^{1-3}$ Majority of these diseases comprise of chronic vascular diseases. ${ }^{4}$ The report on the Bellagio Conference entitled 'prevention of vascular diseases in the emerging world: An approach to global health equity' identifies the menace of chronic vascular diseases around the globe. ${ }^{1}$ On the one hand, the increasing incidence of coronary artery disease (CAD) has become a potential time-bomb causing deaths in countries where preventive measures have not been effective in spite of the excellent in-hospital care; on the other hand, in a resource-poor country like ours, early diagnosis and proper management of cases of acute coronary syndrome (ACS) have still continued to be a challenging task. The lack of awareness about the gravity of consequences of chest pain on the part of the

\footnotetext{
Correspondence:

Dr. Pramod Acharya

Department of Internal Medicine

BPKIHS, Dharan, Nepal.

Email: acharya.pramod@gmail.com
} 
Acharya al. Delayed Presentation of Acute Coronary Syndrome: A Challenge in its Early Management

patients causes a delayed presentation to hospitals. ${ }^{5}$ In the same way, not being able to suspect ACS in the health posts and district hospitals hinders quick referral to a nearby center equipped with the management facility. Prompt and efficient ambulance service is the key to transport such patients. In the present study, we tried to analyze the difficulties in treating ACS in the eastern rural part of the country and find out the causes of delayed presentation to our hospital.

\section{METHODS}

A cross sectional descriptive study was conducted on 100 consecutive patients presenting to B.P. Koirala Institute of Health Sciences (BPKIHS) with ACS over a period of eight months, beginning in June 2005 to February 2006 . Patients who fulfilled the diagnostic criteria for acute ST segment elevation myocardial infarction (STEMI), acute non ST segment elevation myocardial infarction (NSTEMI) and unstable angina (UA) were included. ${ }^{6}$ Those patients who had an alternate diagnosis for the chest pain were excluded. The study was approved by the ethical committee of our institution. After taking informed consent from the subjects, a questionnaire was filled in for each patient, which included demographic and clinical parameters as well the delay and its cause in bringing the patient to our hospital. Data were analyzed with SPSS 11.5 for Windows software.

\section{RESULTS}

The age of the patients ranged from 36 to 84 years (mean age 62 years, SD 10.4). Eighteen patients were of age equal or less than 50 years. Male to female ratio was $1.6: 1$. Most of the patients $(81 \%)$ presented with chest pain while shortness of breath was observed in $49 \%$ of patients. The time of presentation from the onset of symptoms to the healthcare facility ranged from 1- 360 hours (mean 32 hours). Out of 100 patients, 34 patients were residents of Dharan city and 32 of them presented to our institute within 20 hours (mean value). Twenty patients who came to us directly from places outside Dharan had a mean time of presentation within 63 hours (mean value). Similarly, the lapse of time from the onset of symptoms to arrival at a healthcare facility for patients who did not come to our institute directly was 39 hours (mean value). Male patients were more likely to present earlier (29 hours, mean value) than their female counterparts (39 hours, mean value).

Out of 48 patients who came to BPKIHS after attending the local healthcare facility, only 22 patients were actually referred while the remaining 26 patients came by their wish. Eleven of these patients did not try to come to BPKIHS at the onset of symptoms because they thought the symptoms were minor. Nevertheless, they did visit their local health posts or district hospitals or private clinics.

\section{DISCUSSION}

The burden of cardiovascular disease in the Indian subcontinent is quite large and is on the rise. It will become the prevailing overall cause of mortality among the inhabitants of south Asia in the next 20 years. ${ }^{7,8}$ In the present study, the incidence of ACS presenting to our coronary care unit uncovers only the tip of the iceberg. A larger community based study on the presence of CAD and its risk factors can demonstrate the bigger picture. Another major concern is the early age at presentation. Though our study is not a community study to observe the presence of CAD in young, we had $18 \%$ of patients with age equal or less than 50. It has been shown that ischemic heart disease manifests almost 10 years earlier on average in the Indian subcontinent compared with the rest of the world. $^{9-11}$ Smoking and dyslipidemia are known to be the most important risk factors in premature CAD. ${ }^{12}$ Addressing to these risk factors can decrease the life threatening complications of ACS.

Transportation problem is the leading cause of delayed presentation in our study. Presently BPKIHS does not operate any ambulance service on its own. Patients are brought to our hospital by private ambulances and other private vehicles. Patients would greatly benefit if we are equipped with the required number of ambulances of our own. A telephonic conversation with the primary healthcare center can identify the cases and enable us to send an ambulance promptly. This needs an effort both from the doctors in the primary healthcare center as well as the physicians at our emergency department. An effective communication can also reduce the unnecessary delay at the primary healthcare center which was the second most common cause of late arrival.

Electrocardiogram (ECG) is not routinely performed in the district hospitals, health posts and private clinics in patients presenting with chest pain (Table 1). We could not establish the reason why it was not being done. It is likely that either they do not have ECG machine or the doctors were not suspecting ACS in these patients.

Twenty three cases out of 35 cases of STEMI presented more than 24 hours from the onset of chest pain (Table 2). This hindered the administration of thrombolytic agents in our institute and resulted in sub-optimal treatment.

\section{CONCLUSIONS}

There is a strong need to pay attention to the rise of CAD and resulting ACS in our country. New health policies should be made addressing this issue. ${ }^{13}$ Health awareness programs regarding the cause and consequences of chest pain should be conducted in the urban as well as the rural areas. Early diagnosis 


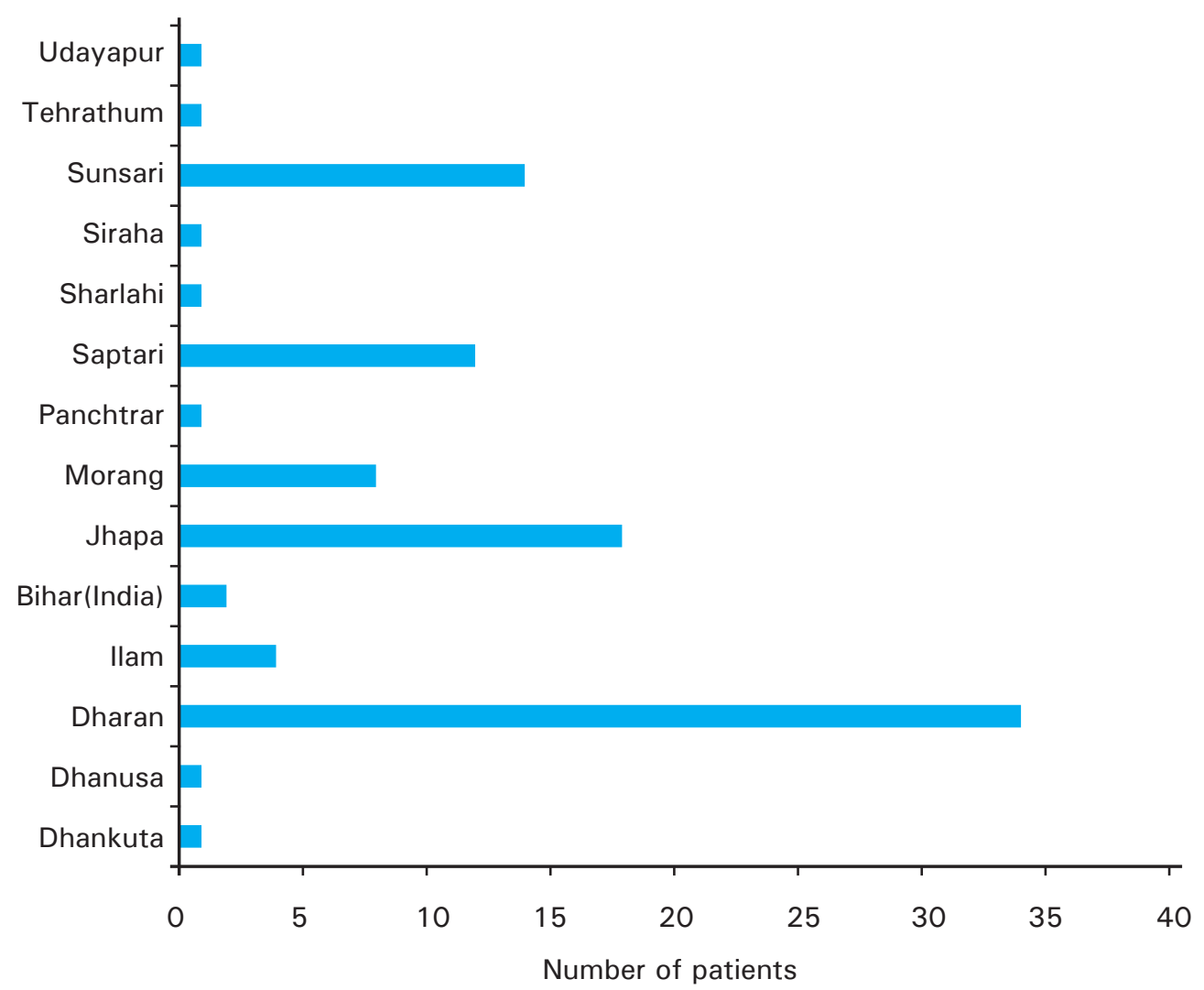

\section{Graph 1. Geographical distribution of patients}

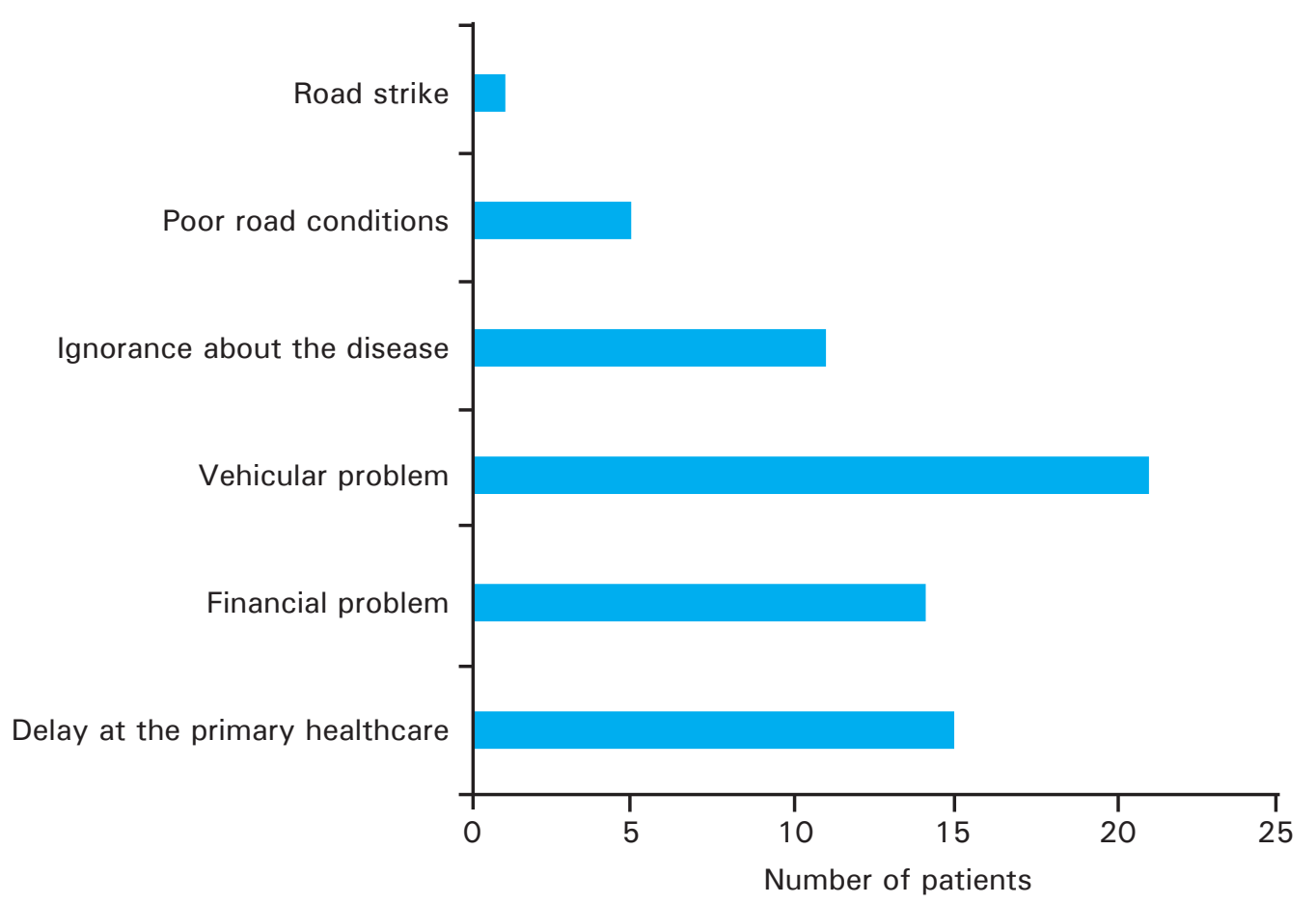

Graph 2: Causes of delay in reaching BPKIHS 
Acharya al. Delayed Presentation of Acute Coronary Syndrome: A Challenge in its Early Management

Table 1. Comparison of various investigations done and therapies given in different healthcare setups before referring to BPKIHS.

\begin{tabular}{lccc}
\hline Investigations done & $\begin{array}{c}\text { District hospitals } \\
\text { Or therapies given }\end{array}$ & $\begin{array}{c}\text { Health posts } \\
(\mathbf{N}=\mathbf{3})\end{array}$ & $\begin{array}{c}\text { Private clinics } \\
\text { (N=23) }\end{array}$ \\
\hline ECG & 15 & 0 & 12 \\
Cardiac enzymes & 0 & 0 & 1 \\
Aspirin & 14 & 2 & 9 \\
Beta-blockers & 1 & 2 & 3 \\
Nitrates & 14 & 2 & 11 \\
Calcium channel blockers & 0 & 0 & 1 \\
Heparin / low molecular weight heparin & 0 & 0 & 0 \\
Thrombolysis & 0 & 0 & 0 \\
Diagnosis of ACS made & 13 & 1 & 7 \\
\hline
\end{tabular}

Table 2. Distribution of acute coronary syndrome components and their hemodynamic profiles in the patient population.

\begin{tabular}{lllll}
\hline Diagnosis & No of patients & SBP & DBP & Pulse \\
\hline STEMI presenting $>24$ hours & 23 & $124(34)$ & $86(21)$ & $80(21)$ \\
STEMI presenting $<24$ hours & 12 & $139(36)$ & $92(24)$ & $78(18)$ \\
NSTEMI & 24 & $134(35)$ & $88(25)$ & $91(24)$ \\
UA & 41 & $130(25)$ & $85(17)$ & $85(21)$ \\
\hline
\end{tabular}

STEMI: ST segment elevation MI, NSTEMI: Non ST segment elevation MI, UA: unstable angina, SBP: Systolic $\mathrm{BP}$ in $\mathrm{mmHg}$ expressed as mean value (standard deviation), DBP: Diastolic BP in $\mathrm{mmHg}$ expressed as mean value (standard deviation), Pulse: Pulse rate per minute (standard deviation)

and effective treatment is the key to reduce morbidity and mortality. Equipping all the district hospitals, health posts and private clinics with an ECG machine, at the least, is recommended. Ambulance services need to be improved.
It is time we collaborate with international organization in various preventive programs and researches. ${ }^{14}$ Strategic plans to prevent chronic vascular diseases are the keys to an effective approach to global health equity. ${ }^{15}$

\section{REFERENCES}

1. Dirks JH, Robinson SW, Alderman M, et al. Meeting report on the Bellagio Conference 'prevention of vascular disease in the emerging world: An approach to global health equity'. Kidney International 2006;70:1397-1402.

2. Reddy KS. Cardiovascular disease in non-western countries. N Engl J Med 2004;350:2438-40.

3. Enas EA, Yusuf S, Mehta JL. Prevalence of coronary artery disease in Asian Indians. Am J Cardiol 1992;70:945-9.

4. Ghaffar A, Reddy KS, Singhi M. Burden of non-communicable diseases in South Asia. BMJ 2004;328:807-10.

5. Albarrran JW, Clarke BA, Crawford J. 'It was not chest pain really, I can't explain it!' An exploratory study on the nature of symptoms experienced by women during their myocardial infarction. J Clin Nurs 2007;16(7):1292-301.

6. Luepker RV, Apple FS, Christenson RH, et al. Case definitions for acute coronary disease in epidemiology and clinical research studies. Circulation 2003; 108: 2543

7. Goyal A, Yusuf S. The burden of cardiovascular disease in the Indian subcontinent. Indian J Med Res 2006;124:235-244.

8. Gupta R. Burden of coronary heart disease in India. Indian Heart J 2005;57:632-8.
9. Yusuf S, Hawken S, Ounpuu S, et al. Effects of potentially modifiable risk factors associated with myocardial infarction in 52 countries (the INTERHEART study): case control study. Lancet 2004;364:937-52.

10. Tewari S, Kumar S, Kapoor A, et al. Premature coronary artery disease in North India: an angiographic study of 1971 patients. Indian Heart Journal 2005;57(4):311- 8.

11. Kearney PM, Whelton M, Reynolds K, et al. Global burden of hypertension: analysis of world wide data. Lancet 2005;365:217-23.

12. MedisS, YachD, Bengoa R, etal. Research gapincardiovascular diseases in developing countries. Lancet 2003;361:2246-2247.

13. Ranjith N, Pegoraro RJ, Naidoo DP. Demographic data and outcome of acute coronary syndrome in South African Asian Indian population. Cardiovasc J S Afr 2005;16:48-54.

14. Ranjith N, Verho NK, Verho M, et al. Acute myocardial infarction in a young South African Indian-based population: patient characteristics on admission and gender-specific risk factor prevalence. Curr Med Res Opin 2002;18:242-8.

15. Reddy KS, Shah B, Varghese C, et al. Responding to the threat of chronic diseases in India. Lancet 2005;366:1746-51. 